\title{
$\lambda$-Analogues of Stirling polynomials of the first kind and their applications
}

Taekyun Kim ${ }^{1,2}$, Dae San Kim ${ }^{3}$, Han Young Kim² and Sung-Soo Pyo ${ }^{4^{*}}$

${ }^{\text {*Correspondence: }}$

ssoopyo@gmail.com

${ }^{4}$ Department of Mathematics

Education, Silla University, Busan,

Republic of Korea

Full list of author information is

available at the end of the article

\begin{abstract}
Recently, $\lambda$-analogues of Stirling numbers of the first kind were studied. In this paper, we introduce, as natural extensions of these numbers, $\lambda$-Stirling polynomials of the first kind and $r$-truncated $\lambda$-Stirling polynomials of the first kind. We give recurrence relations, explicit expressions, some identities, and connections with other special polynomials for those polynomials. Further, as applications, we show that both of them appear in an expression of the probability mass function of a suitable discrete random variable, constructed from $\lambda$-logarithmic and negative $\lambda$-binomial distributions.
\end{abstract}

MSC: 11B73; 11B83; 60G50

Keywords: $\lambda$-Stirling polynomials of the first kind; $r$-truncated $\lambda$-Stirling polynomials of the first kind; $r$-truncated $\lambda$-logarithmic distribution; Negative $\lambda$-binomial distribution

\section{Introduction}

As is known, the Stirling numbers of the first kind are defined by

$$
(x)_{n}=\sum_{l=0}^{n} S_{1}(n, l) x^{l} \quad(n \geq 0)(\text { see }[1,2,4,7-25]),
$$

where $(x)_{0}=1,(x)_{n}=x(x-1) \cdots(x-n+1)(n \geq 1)$.

For any real number $\lambda$, the $\lambda$-analogue of $(x)_{n}$ is defined as

$$
(x)_{0, \lambda}=1, \quad(x)_{n, \lambda}=x(x-\lambda)(x-2 \lambda) \cdots(x-(n-1) \lambda) \quad(n \geq 1) .
$$

Note that $\lim _{\lambda \rightarrow 1}(x)_{n, \lambda}=(x)_{n}(n \geq 0)$ (see $\left.[2,11,13,16,19]\right)$.

The $\lambda$-analogues of the Stirling numbers of the first kind are given by

$$
(x)_{n, \lambda}=\sum_{k=0}^{n} S_{1, \lambda}(n, k) x^{k} \quad(\text { see }[13]) .
$$

We recall that the $\lambda$-binomial coefficients are defined by the generating function

$$
(1+\lambda t)^{\frac{x}{\lambda}}=\sum_{l=0}^{\infty}\left(\begin{array}{l}
x \\
l
\end{array}\right)_{\lambda} t^{l}=\sum_{l=0}^{\infty}(x)_{l, \lambda} \frac{t^{l}}{l !} \quad(\text { see }[13,16]) .
$$

(c) The Author(s) 2019. This article is distributed under the terms of the Creative Commons Attribution 4.0 International License (http://creativecommons.org/licenses/by/4.0/), which permits unrestricted use, distribution, and reproduction in any medium, provided you give appropriate credit to the original author(s) and the source, provide a link to the Creative Commons license, and indicate if changes were made. 
From (4), we note that

$$
\left(\begin{array}{l}
n \\
k
\end{array}\right)_{\lambda}=\frac{(n !)_{\lambda}}{k !(n-k \lambda)_{n-k, \lambda}}=\frac{(n)_{k, \lambda}}{k !} \quad(n \geq k \geq 0)
$$

where

$$
(n !)_{\lambda}=n(n-\lambda)(n-2 \lambda) \cdots(n-(n-1) \lambda)=(n)_{n, \lambda} \quad(\text { see }[13])
$$

and

$$
\sum_{m=0}^{n}\left(\begin{array}{c}
y \\
m
\end{array}\right)_{\lambda}\left(\begin{array}{c}
x \\
n-m
\end{array}\right)_{\lambda}=\left(\begin{array}{c}
x+y \\
n
\end{array}\right)_{\lambda} \quad(n \geq 0)
$$

For $r \in \mathbb{N}$, the unsigned $r$-Stirling numbers of the first kind are defined by

$$
(x+r)(x+r+1) \cdots(x+r+n-1)=\sum_{k=0}^{n}\left[\begin{array}{l}
n+r \\
k+r
\end{array}\right]_{r} x^{k} \quad(\text { see }[10,13,16]) .
$$

In $[16,18]$, the $r$-Stirling numbers of the first kind are given by

$$
(x+r)_{n}=\sum_{k=0}^{n} S_{1}^{(r)}(n, k) x^{k} \quad(n \geq 0) .
$$

By (7) and (8), we get

$$
S_{1}^{(-r)}(n, k)=(-1)^{n-k}\left[\begin{array}{l}
n+r \\
k+r
\end{array}\right]_{r} \quad(n \geq k \geq 0)
$$

It is known that $\lambda$-analogues of $r$-Stirling numbers of the first kind are given by

$$
(x+r)_{n, \lambda}=\sum_{k=0}^{n} S_{1, \lambda}^{(r)}(n, k) x^{k} \quad(\text { see }[16])
$$

From (3), (6), and (10), we note that

$$
S_{1, \lambda}^{(r)}(n, k)=\sum_{m=k}^{n}\left(\begin{array}{c}
n \\
m
\end{array}\right) S_{1, \lambda}(m, k)(r)_{n-m, \lambda} \quad(n, k \geq 0) .
$$

If $X$ is a discrete random variable taking values in the nonnegative integers, then the probability generating function of $X$ is defined as follows:

$$
G(t)=E\left[t^{X}\right]=\sum_{x=0}^{\infty} p(x) t^{x} \quad(\text { see }[7,24])
$$

where $p(x)$ is the probability mass function of $X$. 
Let $X=\left(X_{1}, X_{2}, \ldots, X_{k}\right)$ be a discrete random variable taking values in the $k$-dimensional nonnegative integer lattice. Then the probability generating function of $X$ is defined as follows:

$$
\begin{aligned}
G(t) & =G\left(t_{1}, t_{2}, \ldots, t_{k}\right)=E\left[t_{1}^{X_{1}}, t_{2}^{X_{2}}, \ldots, t_{k}^{X_{k}}\right] \\
& =\sum_{x_{1}, x_{2}, \ldots, x_{k}=0}^{\infty} p\left(x_{1}, x_{2}, \ldots, x_{k}\right) t_{1}^{x_{1}} t_{2}^{x_{2}} \cdots t_{k}^{x_{k}},
\end{aligned}
$$

where $p\left(x_{1}, x_{2}, \ldots, x_{k}\right)$ is the probability mass function of $X$. The power series converges absolutely at least for all convex vectors $t=\left(t_{1}, t_{2}, \ldots, t_{k}\right) \in \mathbb{C}^{k}$ with $\max \left\{\left|t_{1}\right|,\left|t_{2}\right|, \ldots,\left|t_{k}\right|\right\} \leq 1$.

The logarithmic random variable $X$ with parameter $\alpha \in(0,1)$ is a discrete random variable on $\mathbb{N}$ with probability mass function $p(x)$ given by

$$
P[X=n]=p(n)=-\frac{1}{\log (1-\alpha)} \cdot \frac{\alpha^{n}}{n} \quad(n \in \mathbb{N}) .
$$

Note that

$$
\sum_{n=1}^{\infty} p(n)=-\frac{1}{\log (1-\alpha)} \sum_{n=1}^{\infty} \frac{\alpha^{n}}{n}=1
$$

and

$$
E[X]=\sum_{n=1}^{\infty} p(n) \cdot n=-\frac{1}{\log (1-\alpha)} \sum_{n=1}^{\infty} \alpha^{n}=-\frac{1}{\log (1-\alpha)} \cdot \frac{\alpha}{1-\alpha} .
$$

In probability and statistics, the logarithmic distribution (also known as the logarithmic series distribution) is a discrete probability distribution derived from the Maclaurin series expansion

$$
-\log (1-\alpha)=\alpha+\frac{\alpha^{2}}{2}+\frac{\alpha^{3}}{3}+\cdots
$$

In probability theory and statistics, the negative binomial distribution is a discrete probability distribution of the number of successes in a sequence of independent and identically distributed Bernoulli trials before a specified number of failures (denoted by $r$ ) occurs. The negative binomial random variable is sometimes defined in terms of the random variable $Y=$ the number of failures before the $r$ th success. The probability mass function of the negative binomial random variable with parameters $r$ and $p$ is given by

$$
P[X=y]=p(y)=\left(\begin{array}{c}
y+r-1 \\
y
\end{array}\right) p^{y}(1-p)^{r} .
$$

In this paper, we consider $\lambda$-Stirling polynomials of the first kind and truncated $\lambda$-Stirling polynomials of the first kind rising from the $\lambda$-analogues of the falling factorial sequence and investigate some properties for these polynomials. In particular, we give some identities, recurrence relations, and explicit expressions for the $\lambda$-Stirling polynomials of the first kind and the truncated $\lambda$-Stirling polynomials of the first kind. Further, we show 
that both of them appear in an expression of the probability mass function of a suitable discrete random variable, constructed from $\lambda$-logarithmic and negative $\lambda$-binomial distributions.

\section{$2 \lambda$-Stirling polynomials of the first kind}

Let $t$ be a real variable, $x$ be a real number, and let $n$ be a nonnegative integer. The Taylor expansion of the function $(t)_{n, \lambda}$ is given by

$$
(t)_{n, \lambda}=\sum_{k=0}^{\infty} \frac{1}{k !}\left[\frac{d^{k}}{d t^{k}}(t)_{n, \lambda}\right]_{t=x}(t-x)^{k}
$$

Let

$$
S_{1, \lambda}^{(x)}(n, k)=\frac{1}{k !}\left[\frac{d^{k}}{d t^{k}}(t)_{n, \lambda}\right]_{t=x}(n, k \geq 0) .
$$

Then, by (16) and (17), we get

$$
(t)_{n, \lambda}=\sum_{k=0}^{n} S_{1, \lambda}^{(x)}(n, k)(t-x)^{k} \quad(n \geq 0) .
$$

Here $S_{1, \lambda}^{(x)}(n, k)$ will be called the $\lambda$-Stirling polynomials of the first kind.

It is easy to show that

$$
(t)_{n+1, \lambda}=(t-x)(t)_{n, \lambda}+(x-n \lambda)(t)_{n, \lambda} .
$$

From (18), we can derive the following equation:

$$
\begin{aligned}
\sum_{k=0}^{n+1} S_{1, \lambda}^{(x)}(n+1, k)(t-x)^{k} & =(t)_{n+1, \lambda}=(t-x)(t)_{n, \lambda}+(x-n \lambda)(t)_{n, \lambda} \\
& =\sum_{k=0}^{n} S_{1, \lambda}^{(x)}(n, k)(t-x)^{k+1}+(x-n \lambda) \sum_{k=0}^{n} S_{1, \lambda}^{(x)}(n, k)(t-x)^{k} \\
& =\sum_{k=1}^{n+1} S_{1, \lambda}^{(x)}(n, k-1)(t-x)^{k}+(x-n \lambda) \sum_{k=0}^{n} S_{1, \lambda}^{(x)}(n, k)(t-x)^{k} \\
& =\sum_{k=0}^{n+1}\left(S_{1, \lambda}^{(x)}(n, k-1)+(x-n \lambda) S_{1, \lambda}^{(x)}(n, k)\right)(t-x)^{k} .
\end{aligned}
$$

Therefore, by comparing the coefficients on both sides of (20), we obtain the following theorem.

Theorem 2.1 For $n, k \geq 0$ with $n \geq k-1$, we have

$$
S_{1, \lambda}^{(x)}(n, k-1)+(x-n \lambda) S_{1, \lambda}^{(x)}(n, k)=S_{1, \lambda}^{(x)}(n+1, k) .
$$


Note that

$$
S_{1, \lambda}^{(x)}(0,0)=1, \quad S_{1, \lambda}^{(x)}(n, 0)=(x)_{n, \lambda}, \quad S_{1, \lambda}^{(x)}(0, k)=0 \quad(k>0) .
$$

From (18), we easily note that $S_{1, \lambda}^{(x)}(n, k)=0$ if $k>n$. Let us take $t=x+1$ and $t=x-1$ in (18). Then we have

$$
(x+1)_{n, \lambda}=\sum_{k=0}^{n} S_{1, \lambda}^{(x)}(n, k)(x+1-x)^{k}=\sum_{k=0}^{n} S_{1, \lambda}^{(x)}(n, k)
$$

and

$$
(x-1)_{n, \lambda}=\sum_{k=0}^{n} S_{1, \lambda}^{(x)}(n, k)(-1)^{k} .
$$

By (3), we get

$$
\begin{aligned}
\frac{1}{k !}\left[\frac{d^{k}}{d t^{k}}(t)_{n, \lambda}\right]_{t=x} & =\left.\frac{1}{k !} \sum_{l=k}^{n} S_{1, \lambda}(n, l)(l)_{k} t^{l-k}\right|_{t=x} \\
& =\sum_{l=k}^{n} S_{1, \lambda}(n, l)\left(\begin{array}{l}
l \\
k
\end{array}\right) x^{l-k} .
\end{aligned}
$$

From (17) and (21), we obtain the following theorem.

Theorem 2.2 For $n \geq k$, we have

$$
S_{1, \lambda}^{(x)}(n, k)=\sum_{l=k}^{n} S_{1, \lambda}(n, l)\left(\begin{array}{l}
l \\
k
\end{array}\right) x^{l-k}
$$

Note that $S_{1, \lambda}^{(0)}(n, k)=S_{1, \lambda}(n, l)$.

Now, we give an explicit expression for the polynomials $S_{1, \lambda}^{(x)}(n, k)$ and their relations with $\lambda$-Stirling numbers of the first kind. First we observe that

$$
\begin{aligned}
(1+\lambda t)^{\frac{y}{\lambda}} & =\sum_{k=0}^{\infty}(y)_{k, \lambda} \frac{t^{k}}{k !} \\
& =\sum_{k=0}^{\infty}\left(\sum_{n=0}^{k} \frac{1}{n !}\left[\frac{d^{n}}{d y^{n}}(y)_{k, \lambda}\right]_{y=x}(y-x)^{n}\right) \frac{t^{k}}{k !} \\
& =\sum_{n=0}^{\infty}\left(\sum_{k=n}^{\infty} S_{1, \lambda}^{(x)}(k, n) \frac{t^{k}}{k !}\right)(y-x)^{n} .
\end{aligned}
$$


On the other hand,

$$
\begin{aligned}
(1+\lambda t)^{\frac{y}{\lambda}} & =e^{\frac{y}{\lambda} \log (1+\lambda t)} \\
& =\sum_{k=0}^{\infty} \frac{1}{k !}\left(\frac{1}{\lambda} \log (1+\lambda t)\right)^{k} e^{\frac{x}{\lambda} \log (1+\lambda t)}(y-x)^{k} \\
& =\sum_{k=0}^{\infty} \frac{1}{k !}\left(\frac{1}{\lambda} \log (1+\lambda t)\right)^{k}(1+\lambda t)^{\frac{x}{\lambda}}(y-x)^{k}
\end{aligned}
$$

From (22) and (23), we obtain the generating function for $S_{1, \lambda}^{(x)}(n, k)$ given by

$$
\frac{1}{k !}\left(\frac{\log (1+\lambda t)}{\lambda}\right)^{k}(1+\lambda t)^{\frac{x}{\lambda}}=\sum_{n=k}^{\infty} S_{1, \lambda}^{(x)}(n, k) \frac{t^{n}}{n !},
$$

where $k$ is a nonnegative integer.

Indeed, we note that

$$
\begin{aligned}
\frac{1}{k !}\left(\frac{\log (1+\lambda t)}{\lambda}\right)^{k}(1+\lambda t)^{\frac{x}{\lambda}} & =\sum_{m=0}^{\infty}(x)_{m, \lambda} \frac{t^{m}}{m !}\left(\frac{1}{k !} \sum_{l=k}^{\infty}\left(\sum_{l_{1}+l_{2}+\cdots+l_{k}=l} \frac{(-\lambda)^{l-k}}{l_{1} l_{2} \cdots l_{k}}\right) t^{l}\right) \\
& =\sum_{n=k}^{\infty}\left(\frac{1}{k !} \sum_{l=k}^{n}(-\lambda)^{l-k} l !\left(\begin{array}{c}
n \\
l
\end{array}\right)(x)_{n-l, \lambda} \sum_{l_{1}+l_{2}+\cdots+l_{k}=l} \frac{1}{l_{1} l_{2} \cdots l_{k}}\right) \frac{t^{n}}{n !}
\end{aligned}
$$

Therefore, by (24) and (25), we obtain the following theorem.

Theorem 2.3 For $n \geq k$, we have

$$
S_{1, \lambda}^{(x)}(n, k)=\frac{1}{k !} \sum_{l=k}^{n}(-\lambda)^{l-k} l !\left(\begin{array}{c}
n \\
l
\end{array}\right)(x)_{n-l, \lambda} \sum_{l_{1}+l_{2}+\cdots+l_{k}=l} \frac{1}{l_{1} l_{2} \cdots l_{k}},
$$

where the inner sum runs over all positive integers $l_{1}, l_{2}, \ldots, l_{k}$ with $l_{1}+l_{2}+\cdots+l_{k}=l$.

It is known that

$$
\frac{1}{k !}\left(\frac{1}{\lambda} \log (1+\lambda t)\right)^{k}=\sum_{n=k}^{\infty} S_{1, \lambda}(n, k) \frac{t^{n}}{n !} \quad(\text { see }[13,16]) .
$$

From (24) and (26), we have

$$
\begin{aligned}
\sum_{n=k}^{\infty} S_{1, \lambda}^{(x)}(n, k) \frac{t^{n}}{n !} & =\sum_{l=0}^{\infty}(x)_{l, \lambda} \frac{t^{l}}{l !} \sum_{m=k}^{\infty} S_{1, \lambda}(m, k) \frac{t^{m}}{m !} \\
& =\sum_{n=k}^{\infty}\left(\sum_{l=0}^{n-k}(x)_{l, \lambda}\left(\begin{array}{c}
n \\
l
\end{array}\right) S_{1, \lambda}(n-l, k)\right) \frac{t^{n}}{n !} \\
& =\sum_{n=k}^{\infty}\left(\sum_{l=k}^{n}(x)_{n-l, \lambda}\left(\begin{array}{c}
n \\
l
\end{array}\right) S_{1, \lambda}(l, k)\right) \frac{t^{n}}{n !}
\end{aligned}
$$


Comparing the coefficients on both sides of (27), we obtain the following theorem.

Theorem 2.4 Let $n, k$ be nonnegative integers. Then we have

$$
S_{1, \lambda}^{(x)}(n, k)= \begin{cases}\sum_{l=k}^{n}\left(\begin{array}{l}
n \\
l
\end{array}\right)(x)_{n-l, \lambda} S_{1, \lambda}(l, k), & \text { if } k \leq n, \\
0, & \text { if } k>n .\end{cases}
$$

Corollary 2.5 Let $n, k$ be nonnegative integers. Then we have

$$
S_{1, \lambda}(n, k)= \begin{cases}\sum_{l=k}^{n}\left(\begin{array}{l}
n \\
l
\end{array}\right)(x)_{n-l, \lambda} S_{1, \lambda}^{(x)}(l, k), & \text { if } k \leq n \\
0, & \text { if } k>n\end{cases}
$$

We now consider the $r$-truncated $\lambda$-Stirling numbers of the first kind.

For $x \in \mathbb{R}$ and $r \in \mathbb{N}$, the $r$-truncated $\lambda$-Stirling polynomials of the first kind are defined by

$$
(1+\lambda t)^{\frac{x}{\lambda}} \frac{1}{k !}\left(\frac{\log (1+\lambda t)}{\lambda}-\sum_{j=1}^{r-1}(-\lambda)^{j-1} \frac{t^{j}}{j}\right)^{k}=\sum_{n=r k}^{\infty} S_{1, \lambda}^{(x)}(n, k \mid r) \frac{t^{n}}{n !} .
$$

Remark 2.6 The definition of $\lambda$-Stirling polynomials of the first kind and that of $r$ truncated $\lambda$-Stirling polynomials of the first kind are similar to the non-central Stirling numbers of the first kind and the generalized non-central Stirling numbers of the first kind, respectively (see [20]). In fact, one replaces $\alpha$ by $x$ and $(x)_{n}$ by $(x)_{n, \lambda}$, as in [3] and [6], or replaces $\alpha$ by $x$ with setting $\alpha_{i}=i \lambda, i=0,1, \ldots, n-1$, as in (1.8) of [5].

From (28), we have

$$
\begin{aligned}
(1+\lambda t)^{\frac{x}{\lambda}} \frac{1}{k !}\left(\frac{\log (1+\lambda t)}{\lambda}-\sum_{j=1}^{r-1}(-\lambda)^{j-1} \frac{t^{j}}{j}\right)^{k} \\
=\sum_{m=0}^{\infty}(x)_{m, \lambda} \frac{t^{m}}{m !} \frac{1}{k !} \sum_{l=r k}^{\infty}\left(\sum_{l_{1}+l_{2}+\cdots+l_{k}=l} \frac{(-\lambda)^{l-k}}{l_{1} l_{2} \cdots l_{k}}\right) t^{l} \\
=\sum_{n=k r}^{\infty}\left(\frac{n !}{k !} \sum_{l=r k}^{n} \frac{(x)_{n-l, \lambda}}{(n-l) !} \sum_{l_{1}+\cdots+l_{k}=l} \frac{(-\lambda)^{l-k}}{l_{1} l_{2} \cdots l_{k}}\right) \frac{t^{n}}{n !} .
\end{aligned}
$$

Therefore, by (28) and (29), we obtain the following theorem.

Theorem 2.7 For $n \geq r k$, we have

$$
S_{1, \lambda}^{(x)}(n, k \mid r)=\frac{n !}{k !} \sum_{l=r k}^{n} \frac{(x)_{n-l, \lambda}}{(n-l) !} \sum_{l_{1}+\cdots+l_{k}=l} \frac{(-\lambda)^{l-k}}{l_{1} l_{2} \cdots l_{k}} .
$$

In particular, if $n<k r$, we have

$$
S_{1, \lambda}^{(x)}(n, k \mid r)=0 .
$$


When $x=0, S_{1, \lambda}^{(0)}(n, k \mid r)=S_{1, \lambda}(n, k \mid r)$ are called the $r$-truncated $\lambda$-Stirling numbers of the first kind.

It is not difficult to show that

$$
S_{1, \lambda}^{(x)}(n, k \mid r)=\sum_{l=k r}^{n}\left(\begin{array}{l}
n \\
l
\end{array}\right)(x)_{n-l, \lambda} S_{1, \lambda}(l, k \mid r) \quad \text { if } n \geq k r
$$

and

$$
S_{1, \lambda}^{(x)}(n, k \mid r)=0 \quad \text { if } n<k r .
$$

Let

$$
\left.y_{k, \lambda}^{(r)}(t \mid x)=(1+\lambda t)^{\frac{x}{\lambda}} \frac{1}{k !}\left[\frac{1}{\lambda} \log 1+\lambda t\right)-\sum_{j=1}^{r-1} \frac{(-\lambda)^{j-1}}{j} t^{j}\right]^{k} .
$$

From (30), we can derive the following differential equation:

$$
(1+\lambda t) \frac{d}{d t} y_{k, \lambda}^{(r)}(t \mid x)=x y_{k, \lambda}^{(r)}(t \mid x)+(-1)^{r-1} t^{r-1} \lambda^{r-1} y_{k-1, \lambda}^{(r)}(t \mid x) .
$$

By (28) and (31), we get

$$
\begin{aligned}
(1 & +\lambda t) \frac{d}{d t} y_{k, \lambda}^{(r)}(t \mid x) \\
& =x y_{k, \lambda}^{(r)}(t \mid x)+(-1)^{r-1} t^{r-1} \lambda^{r-1} y_{k-1, \lambda}^{(r)}(t \mid x) \\
& =x \sum_{n=r k}^{\infty} S_{1, \lambda}^{(x)}(n, k \mid r) \frac{t^{n}}{n !}+(-1)^{r-1} t^{r-1} \lambda^{r-1} \sum_{n=r(k-1)}^{\infty} S_{1, \lambda}^{(x)}(n, k-1 \mid r) \frac{t^{n}}{n !} \\
& =x \sum_{n=r k}^{\infty} S_{1, \lambda}^{(x)}(n, k \mid r) \frac{t^{n}}{n !}+(-1)^{r-1} \lambda^{r-1} \sum_{n=r k-1}^{\infty} S_{1, \lambda}^{(x)}(n-r+1, k-1 \mid r) \frac{t^{n}}{(n-r+1) !} \\
& =\sum_{n=r k-1}^{\infty}\left(x S_{1, \lambda}^{(x)}(n, k \mid r)+(-1)^{r-1} \lambda^{r-1}(r-1) !\left(\begin{array}{c}
n \\
r-1
\end{array}\right) S_{1, \lambda}^{(x)}(n-r+1, k-1 \mid r)\right) \frac{t^{n}}{n !} .
\end{aligned}
$$

On the other hand,

$$
\begin{aligned}
(1+\lambda t) \frac{d}{d t} y_{k, \lambda}^{(r)}(t \mid x) & =\sum_{n=r k}^{\infty} S_{1, \lambda}^{(x)}(n, k \mid r) \frac{t^{n-1}}{(n-1) !}(1+\lambda t) \\
& =\sum_{n=r k-1}^{\infty} S_{1, \lambda}^{(x)}(n+1, k \mid r) \frac{t^{n}}{n !}+\sum_{n=r k}^{\infty} n \lambda S_{1, \lambda}^{(x)}(n, k \mid r) \frac{t^{n}}{n !} \\
& =\sum_{n=r k-1}^{\infty}\left(S_{1, \lambda}^{(x)}(n+1, k \mid r)+n \lambda S_{1, \lambda}^{(x)}(n, k \mid r)\right) \frac{t^{n}}{n !}
\end{aligned}
$$

Therefore, by (32) and (33), we obtain the following theorem. 
Theorem 2.8 Let $n, k$ be nonnegative integers, and let $r$ be a positive integer. Then we have

$$
\begin{aligned}
& \lambda^{r-1}(r-1) !\left(\begin{array}{c}
-n+r-2 \\
r-1
\end{array}\right) S_{1, \lambda}^{(x)}(n-r+1, k-1 \mid r) \\
& \quad=S_{1, \lambda}^{(x)}(n+1, k \mid r)+(n \lambda-x) S_{1, \lambda}^{(x)}(n, k \mid r) \quad(n \geq k r-1) .
\end{aligned}
$$

It is easy to show that

$$
\begin{aligned}
y_{k, \lambda}^{(r+1)}(t \mid x) & =(1+\lambda t)^{\frac{x}{\lambda}} \frac{1}{k !}\left[\frac{1}{\lambda} \log (1+\lambda t)-\sum_{j=1}^{r}(-\lambda)^{j-1} \frac{t^{j}}{j}\right]^{k} \\
& =(1+\lambda t)^{\frac{x}{\lambda}} \frac{1}{k !}\left[\frac{1}{\lambda} \log (1+\lambda t)-\sum_{j=1}^{r-1}(-\lambda)^{j-1} \frac{t^{j}}{j}+(-1)^{r} \lambda^{r-1} \frac{t^{r}}{r}\right]^{k} \\
& =(1+\lambda t)^{\frac{x}{\lambda}} \frac{1}{k !} \sum_{l=0}^{k}\left(\begin{array}{l}
k \\
l
\end{array}\right)\left(\frac{1}{\lambda} \log (1+\lambda t)-\sum_{j=1}^{r-1}(-\lambda)^{j-1} \frac{t^{j}}{j}\right)^{k-l}(-1)^{r l} \frac{\lambda^{r l-l}}{r^{l}} t^{r l} \\
& =\sum_{l=0}^{k} \frac{(-1)^{r l} \lambda^{r l-l}}{l ! r^{l}} t^{r l} \frac{1}{(k-l) !}(1+\lambda t)^{\frac{x}{\lambda}}\left(\frac{\log (1+\lambda t)}{\lambda}-\sum_{j=1}^{r-1}(-1)^{j-1} \frac{t^{j}}{j}\right)^{k-l} \\
& =\sum_{l=0}^{k} \frac{(-1)^{r l} \lambda^{r l-l}}{l ! r^{l}} t^{r l} y_{k-l, \lambda}^{(r)}(t \mid x) .
\end{aligned}
$$

By (34), we get

$$
\begin{aligned}
\sum_{n=k r}^{\infty} S_{1, \lambda}^{(x)}(n, k \mid r+1) \frac{t^{n}}{n !} & =\sum_{n=k(r+1)}^{\infty} S_{1, \lambda}^{(x)}(n, k \mid r+1) \frac{t^{n}}{n !} \\
& =\sum_{l=0}^{k} \frac{(-1)^{r l} \lambda^{r l-l}}{l ! r^{l}} t^{r l} \sum_{n=(k-l) r}^{\infty} S_{1, \lambda}^{(x)}(n, k-l \mid r+1) \frac{t^{n}}{n !} \\
& =\sum_{n=k r}^{\infty}\left(\sum_{l=0}^{k} \frac{(-1)^{r l} \lambda^{r l-l}}{l ! r^{l}} S_{1, \lambda}^{(x)}(n-l r, k-l \mid r)(n)_{l r}\right) \frac{t^{n}}{n !} .
\end{aligned}
$$

Comparing the coefficients on both sides of (35), we have

$$
S_{1, \lambda}^{(x)}(n, k \mid r+1)=\sum_{l=0}^{k} \frac{(-1)^{r l} \lambda^{r l-l}}{l ! r^{l}} S_{1, \lambda}^{(x)}(n-l r, k-l \mid r)(n)_{l r},
$$

where $n \geq k r$.

For $\lambda \in(0,1), X$ is a random variable with the $\lambda$-logarithmic distribution with parameter $\alpha \in(0,1)$ if the probability mass function of $X$ is given by

$$
P_{\lambda}[X=k]=P_{\lambda}(k)=-\frac{\lambda}{\log (1-\alpha \lambda)} \cdot \frac{\alpha^{k} \lambda^{k-1}}{k},
$$

where $k$ is a positive integer. 
We easily see that

$$
\sum_{k=1}^{\infty} P_{\lambda}(k)=1, \quad E[X]=-\frac{1}{\log (1-\alpha \lambda)} \cdot \frac{\alpha \lambda}{1-\alpha \lambda} .
$$

$Y$ is the random variable with negative $\lambda$-binomial distribution with parameters $r, \alpha$ if the probability mass function of $Y$ is given by

$$
P_{\lambda}[Y=k]=P_{\lambda}(k)=\left(\begin{array}{c}
\frac{r}{\lambda}+k-1 \\
k
\end{array}\right)(\lambda \alpha)^{k}(1-\lambda \alpha)^{\frac{r}{\lambda}},
$$

where $r, k, \alpha$ are respectively the number of failures, the number of successes, and the probability of successes.

Let $X_{1}, X_{2}, \ldots, X_{k}$ be independent random variables with $\lambda$-logarithmic distribution with parameter $\alpha$, and let $Y$ be the random variable with negative $\lambda$-binomial distribution with parameters $r$ and $\alpha$. If $Y$ is independent of $X=X_{1}+\cdots+X_{k}$, then we have

$$
E\left[t^{X+Y}\right]=E\left[t^{X}\right] E\left[t^{Y}\right]=\left(\prod_{j=1}^{k} E\left[t^{X}\right]\right) \cdot E\left[t^{Y}\right] .
$$

Now, we observe that

$$
E\left[t^{X_{j}}\right]=\sum_{x=1}^{\infty} P_{\lambda}\left[X_{j}=x\right] t^{x}=\frac{1}{\log (1-\alpha \lambda)} \cdot \log (1-\alpha \lambda t)
$$

and

$$
\begin{aligned}
E\left[t^{Y}\right] & =\sum_{y=0}^{\infty} P_{\lambda}[Y=y] t^{y}=\sum_{y=0}^{\infty}\left(\begin{array}{c}
\frac{r}{\lambda}+y-1 \\
y
\end{array}\right)(\lambda \alpha)^{y}(1-\lambda \alpha)^{\frac{r}{\lambda}} t^{y} \\
& =(1-\lambda \alpha)^{\frac{r}{\lambda}}(1-\lambda \alpha t)^{-\frac{r}{\lambda}} .
\end{aligned}
$$

From (38), (39), and (40), we have

$$
\begin{aligned}
E\left[t^{X+Y}\right] & =\left(\prod_{j=1}^{k} E\left[t^{X}\right]\right) \cdot E\left[t^{Y}\right] \\
& =\left(\frac{1}{\log (1-\alpha \lambda)}\right)^{k}(\log (1-\alpha \lambda t))^{k}(1-\alpha \lambda)^{\frac{r}{\lambda}}(1-\alpha \lambda t)^{-\frac{r}{\lambda}} \\
& =k !\left(\frac{\lambda}{\log (1-\alpha \lambda)}\right)^{k}(1-\alpha \lambda)^{\frac{r}{\lambda}} \frac{1}{k !}\left(\frac{\log (1-\alpha \lambda t)}{\lambda}\right)^{k}(1-\alpha \lambda t)^{-\frac{r}{\lambda}} \\
& =k !\left(\frac{\lambda}{\log (1-\alpha \lambda)}\right)^{k}(1-\alpha \lambda)^{\frac{r}{\lambda}} \sum_{n=k}^{\infty} S_{1, \lambda}^{(-r)}(n, k)(-\alpha)^{n} \frac{t^{n}}{n !} .
\end{aligned}
$$

On the other hand,

$$
E\left[t^{X+Y}\right]=\sum_{n=k}^{\infty} P_{\lambda}[X+Y=n] t^{n}
$$


Therefore, by (41) and (42), we obtain the following theorem.

Theorem 2.9 Let $X_{1}, X_{2}, \ldots, X_{k}$ be independent random variables with $\lambda$-logarithmic distribution with parameter $\alpha$, and let $Y$ be the random variable with negative $\lambda$-binomial distribution with parameters $r$ and $\alpha$. If $Y$ is independent of $X=X_{1}+X_{2}+\cdots+X_{k}$, then the probability mass function of $X+Y$ is given by

$$
P_{\lambda}[X+Y=n]=k !\left(\frac{\lambda}{\log (1-\alpha \lambda)}\right)^{k}(1-\alpha \lambda)^{\frac{r}{x}} \frac{(-\alpha)^{n}}{n !} S_{1, \lambda}^{(-r)}(n, k)
$$

for $n \geq k$.

For $r \in \mathbb{N}, X$ is the random variable with $r$-truncated $\lambda$-logarithmic distribution with parameter $\alpha$ if the probability mass function of $X$ is given by

$$
\begin{aligned}
P_{\lambda}[X=x] & =P_{\lambda}(x)=\frac{\lambda}{-\log (1-\alpha \lambda)-\sum_{i=1}^{r-1} \frac{\lambda^{i} \alpha^{i}}{i}} \cdot \frac{\alpha^{x} \lambda^{x-1}}{x} \\
& =C_{\lambda}(\alpha, r) \frac{\alpha^{x} \lambda^{x-1}}{x} \quad(x=r, r+1, \ldots),
\end{aligned}
$$

where

$$
C_{\lambda}(\alpha, r)=\frac{\lambda}{-\log (1-\alpha \lambda)-\sum_{i=1}^{r-1} \frac{\lambda^{i} \alpha^{i}}{i}}
$$

Note that $\sum_{x=r}^{\infty} P_{\lambda}[X=x]=1$.

Let $X_{1}, X_{2}, \ldots, X_{k}$ be independent random variables with the $r$-truncated $\lambda$-logarithmic distribution with parameter $p$, and let $Y$ be the random variable with negative $\lambda$-binomial distribution with parameters $\alpha, p$. If $Y$ is independent of $X=X_{1}+X_{2}+\cdots+X_{k}$, then we have

$$
E\left[t^{X+Y}\right]=E\left[t^{X}\right] E\left[t^{Y}\right]=\left(\prod_{j=1}^{k} E\left[t^{X_{j}}\right]\right) E\left[t^{Y}\right] .
$$

Now, we observe that

$$
\begin{aligned}
E\left[t^{X_{j}}\right] & =\sum_{x=r}^{\infty} P_{\lambda}\left[X_{j}=x\right] t^{x} \\
& =C_{\lambda}(p, r) \sum_{x=r}^{\infty} \frac{\lambda^{x-1} p^{x}}{x} t^{x} \\
& =C_{\lambda}(p, r)\left(\sum_{x=1}^{\infty} \frac{\lambda^{x-1} p^{x}}{x} t^{x}-\sum_{x=1}^{r-1} \frac{\lambda^{x-1} p^{x}}{x} t^{x}\right) \\
& =C_{\lambda}(p, r)\left(-\frac{1}{\lambda} \log (1-\lambda p t)-\sum_{x=1}^{r-1} \frac{\lambda^{x-1} p^{x}}{x} t^{x}\right),
\end{aligned}
$$


and

$$
\begin{aligned}
E\left[t^{Y}\right] & =\sum_{y=0}^{\infty}\left(\begin{array}{c}
\frac{\alpha}{\lambda}+y-1 \\
y
\end{array}\right)(\lambda p)^{y}(1-\lambda p)^{\frac{\alpha}{\lambda}} t^{y} \\
& =(1-\lambda p t)^{-\frac{\alpha}{\lambda}}(1-\lambda p)^{\frac{\alpha}{\lambda}} .
\end{aligned}
$$

From (43), (44), and (45), we have

$$
\begin{aligned}
E\left[t^{X+Y}\right] & =k ! C_{\lambda}(p, r)^{k}(-1)^{k} \frac{1}{k !}\left(\frac{\log (1-\lambda p t)}{\lambda}+\sum_{x=1}^{r-1} \frac{\lambda^{x-1}}{x} p^{x} t^{x}\right)^{k}(1-\lambda p t)^{-\frac{\alpha}{\lambda}}(1-\lambda p)^{\frac{\alpha}{\lambda}} \\
& =k ! C_{\lambda}(p, r)^{k}(1-\lambda p)^{\frac{\alpha}{\lambda}}(-1)^{k} \sum_{n=r k}^{\infty} S_{1, \lambda}^{(-\alpha)}(n, k \mid r) \frac{(-p)^{n}}{n !} t^{n}
\end{aligned}
$$

On the other hand,

$$
E\left[t^{X+Y}\right]=\sum_{n=r k}^{\infty} P_{\lambda}[X+Y=n] t^{n} .
$$

Therefore, by (46) and (47), we obtain the following theorem.

Theorem 2.10 For $r \in \mathbb{N}$, let $X_{1}, X_{2}, \ldots, X_{k}$ be independent random variables with the $r$ truncated $\lambda$-logarithmic distribution with parameter $p$, and let $Y$ be the random variable with negative $\lambda$-binomial distribution with parameters $\alpha, p$. If $Y$ is independent of $X=$ $X_{1}+X_{2}+\cdots+X_{k}$, then the probability mass function of $X+Y$ is given by

$$
P_{\lambda}[X+Y=n]=k ! C_{\lambda}(p, r)^{k}(1-\lambda p)^{\frac{\alpha}{\lambda}}(-1)^{n-k} S_{1, \lambda}^{(-\alpha)}(n, k \mid r) \frac{p^{n}}{n !} \quad(n \geq k r),
$$

where

$$
C_{\lambda}(\alpha, r)=\frac{\lambda}{-\log (1-\alpha \lambda)-\sum_{i=1}^{r-1} \frac{\lambda^{i} \alpha^{i}}{i}} .
$$

\section{Conclusion}

Stirling numbers of the first kind appear frequently in combinatorics and number theory. Recently, $\lambda$-analogues of Stirling numbers of the first kind were studied in [10].

In this paper, we introduced $\lambda$-Stirling polynomials of the first kind which appear as the coefficients in the Taylor expansion of $\lambda$-falling factorial sequence and reduce to the Stirling numbers of the first kind when $x=0$ and $\lambda=1$. We obtained recurrence relations, explicit expressions, some identities, and connections with other special polynomials for these polynomials. We showed that they appear in an expression of the probability mass function of a suitable discrete random variable, constructed from $\lambda$-logarithmic and negative $\lambda$-binomial distributions. Thereby we demonstrated that these polynomials are not out of nowhere but arise naturally.

We also considered $r$-truncated $\lambda$-Stirling polynomials of the first kind whose generating function is obtained from that of the $\lambda$-Stirling polynomials of the first kind by truncating first $r-1$ terms in the Taylor expansion of the logarithmic function. We derived 
several basic properties about these polynomials just in the case of $\lambda$-Stirling polynomials of the first kind. Then we showed that they also appear in an expression of the probability mass function of a suitable discrete random variable, constructed from $r$-truncated $\lambda$-logarithmic and negative $\lambda$-binomial distributions. Once again, this demonstrates that $r$-truncated $\lambda$-Stirling polynomials of the first kind arise naturally.

As one of our future projects, we would like to continue to find many applications of $\lambda$-Stirling polynomials of the first kind and $r$-truncated $\lambda$-Stirling polynomials of the first kind in mathematics, sciences, and engineering.

Acknowledgements

The authors would like to thank the referees for their helpful comments and suggestions.

\section{Funding}

This research was supported by the Basic Science Research Program through the National Research Foundation of Korea (NRF) funded by the Ministry of Education (2018R1D1A1B07049996).

\section{Ethics approval and consent to participate}

All authors reveal that there is no ethical problem in the production of this paper.

\section{Competing interests}

The authors declare that they have no competing interests.

\section{Consent for publication}

All authors want to publish this paper in this journal.

\section{Authors' contributions}

TK and DSK conceived of the framework and structured the whole paper; TK wrote the paper; S-SP and HYK checked the results of the paper; DSK and TK completed the revision of the article. All authors read and approved the final manuscript.

\section{Author details}

${ }^{1}$ School of Science, Xi'an Technological University, Xi'an, People's Republic of China. ${ }^{2}$ Department of Mathematics, Kwangwoon University, Seoul, Republic of Korea. ${ }^{3}$ Department of Mathematics, Sogang University, Seoul, Republic of Korea. ${ }^{4}$ Department of Mathematics Education, Silla University, Busan, Republic of Korea.

\section{Publisher's Note}

Springer Nature remains neutral with regard to jurisdictional claims in published maps and institutional affiliations.

Received: 4 July 2019 Accepted: 13 November 2019 Published online: 21 November 2019

\section{References}

1. Araci, S., Acikgoz, M.: A note on the Frobenius-Euler numbers and polynomials associated with Bernstein polynomials. Adv. Stud. Contemp. Math. (Kyungshang) 22(3), 399-406 (2012)

2. Carlitz, L.: Degenerate Stirling, Bernoulli and Eulerian numbers. Util. Math. 15, 51-88 (1979)

3. Comtet, L.: Nombres de stirling généraux et fonctions symétriques. C. R. Acad. Sci. 275, 747-750 (1972)

4. Dolgy, D.V., Kim, T.: Some explicit formulas of degenerate Stirling numbers associated with the degenerate special numbers and polynomials. Proc. Jangjeon Math. Soc. 21(2), 309-317 (2018)

5. El-Desouky, B.S.: The multiparameter non-central Stirling numbers. Fibonacci Q. 32, 218-225 (1994)

6. El-Desouky, B.S.: A generalization of Stirling, Lah and harmonic numbers with some computational applications. Ars Comb. 130, 333-356 (2017)

7. Feller, W.: An Introduction to Probability Theory and Its Applications, vol. II, 2nd edn. Wiley, New York (1971)

8. He, Y., Pan, J.: Some recursion formulas for the number of derangements and Bell numbers. J. Math. Res. Appl. 36(1), $15-22(2016)$

9. Howard, F.T.: Bell polynomials and degenerate Stirling numbers. Rend. Semin. Mat. Univ. Padova 61, 203-219 (1979)

10. Jeong, J., Rim, S.-H., Kim, B.M.: On finite-times degenerate Cauchy numbers and polynomials. Adv. Differ. Equ. 2015, Article ID 321 (2015)

11. Kim, D.S., Dolgy, D.V., Kim, D., Kim, T.: Some identities on r-central factorial numbers and $r$-central Bell polynomials. Adv. Differ. Equ. 2019, Article ID 245 (2019)

12. Kim, D.S., Kim, T., Jang, G.-W.: A note on degenerate Stirling numbers of the first kind. Proc. Jangjeon Math. Soc. 21(3), 393-404 (2018)

13. Kim, T.: $\boldsymbol{\lambda}$-Analogue of Stirling numbers of the first kind. Adv. Stud. Contemp. Math. (Kyungshang) 27(3), 423-429 (2017)

14. Kim, T., Kim, D.S.: On $\lambda$-Bell polynomials associated with umbral calculus. Russ. J. Math. Phys. 24(1), 69-78 (2017)

15. Kim, T., Kim, D.S.: Extended Stirling numbers of the first kind associated with Daehee numbers and polynomials. Rev. R. Acad. Cienc. Exactas Fís. Nat., Ser. A Mat. 113(2), 1159-1171 (2019)

16. Kim, T., Kim, D.S.: Some identities on $\lambda$-analogues of $r$-Stirling numbers of the first kind. Filomat (in press) 
17. Kim, T., Kim, D.S., Kwon, H.-I.: A note on degenerate Stirling numbers and their applications. Proc. Jangjeon Math. Soc. 21(2), 195-203 (2018)

18. Kim, T., Yao, Y., Kim, D.S., Jang, G.-W.: Degenerate $r$-Stirling numbers and r-Bell polynomials. Russ. J. Math. Phys. 25(1), 44-58 (2018)

19. Kim, T., Yao, Y., Kim, D.S., Kwon, H.-I.: Some identities involving special numbers and moments of random variables. Rocky Mt. J. Math. 49(2), 521-538 (2019)

20. Koutras, M.: Non-central Stirling numbers and some applications. Discrete Math. 42, 73-89 (1982)

21. Park, J.-W., Kim, B.M., Kwon, J.: On a modified degenerate Daehee polynomials and numbers. J. Nonlinear Sci. Appl. 10(3), 1105-1115 (2017)

22. Pyo, S.-S.: Degenerate Cauchy numbers and polynomials of the fourth kind. Adv. Stud. Contemp. Math. (Kyungshang) 28(1), 127-138 (2018)

23. Roman, S.: The Umbral Calculus. Pure and Applied Mathematics, vol. 111. Academic Press, New York (1984)

24. Ross, S.M.: Introduction to Probability Models, 11 th edn. Elsevier, Amsterdam (2014)

25. Simsek, Y.: Identities and relations related to combinatorial numbers and polynomials. Proc. Jangjeon Math. Soc. 20(1), 127-135 (2017)

Submit your manuscript to a SpringerOpen ${ }^{\circ}$ journal and benefit from:

- Convenient online submission

- Rigorous peer review

- Open access: articles freely available online

- High visibility within the field

- Retaining the copyright to your article

Submit your next manuscript at $\gg$ springeropen.com 